\title{
Airpath Strategy for Experimental Transient Control of a Diesel $\mathrm{HCCl}$ Engine
}

\author{
J. Chauvin ${ }^{1}, 2$, G. Corde', N. Petif and P. Rouchon ${ }^{2}$ \\ 1 Institut français du pétrole, IFP, 1-4, avenue de Bois-Préau, 92852 Rueil-Malmaison Cedex - France \\ 2 Centre Automatique et Systèmes, École des Mines de Paris, 60 blvd Saint Michel, 75006 Paris - France \\ e-mail: jonathan.chauvin@ifp.fr - gilles.corde@ifp.fr - peti@@cas.ensmp.fr - rouchon@cas.ensmp.fr
}

\begin{abstract}
Résumé - Contrôle expérimental de la boucle d'air d'un moteur HCCI en transitoire - Une stratégie de contrôle basée sur une planification de trajectoire est proposée pour la commande de la boucle d'air d'un moteur Diesel turbocompressé avec recirculation de gaz d'échappement (EGR). Le modèle considéré utilise des équations physiques simples de conservation de masse et d'énergie. La dynamique entièrement actionnée est facilement inversée, permettant de génerer une loi de commande boucle ouverte. Un feedback est réalisé en utilisant un observateur pour les variables non directement mesurés. Des essais au banc moteur ont été réalisés sur un moteur de 4 cylindres dans le mode de combustion homogène (HCCI). Les conclusions soulignent la possibilité de tenir compte des dynamiques de non-minimum de phase de ce système par une simple loi de commande. Les transitoires réalisés sont précis et rapides.
\end{abstract}

\begin{abstract}
Airpath Strategy for Experimental Transient Control of a Diesel HCCI Engine A motion planning based control strategy is proposed for the airpath control of turbocharged Diesel engines using exhaust gas recirculation (EGR). The considered model uses simple balance equations. The fully actuated dynamics are easily inverted, yielding straightforward open-loop control laws. This approach is complemented by experimentally derived look-up tables to cast the drivers requests into transients between operating points. Estimation of required variables is addressed and experimental tests are reported on a 4-cylinder engine in Homogeneous Charge Compression Ignition (HCCI) mode. Conclusions stress the possibility of taking into account the non-minimum phase e? ects of this system by a simple, yet effcient in practice, control law. Observed transients are accurate and fast.
\end{abstract}




\section{INTRODUCTION}

Increasingly stringent pollution standards norms have spurred a broad interest in the reduction of global engine emissions. Lately, two strategies have emerged: aftertreatment and direct combustion emissions reduction. For Diesel engines, equipments required by after-treatment and implementation issues usually carry high cost premiums. An alternative is to use a cleaner combustion mode. Therefore, the Highly Premixed Combustion mode (HPC) - including Homogeneous Charge Compression Ignition (HCCI) - has become of major interest in recent years. It requires the use of high Exhaust Gas Recirculation (EGR) rates. The key idea is that the inert burnt gas in the cylinder lower the temperature and dilute the air charge which reduce the emissions of nitrogen oxides. In practice, numerous experimentations brought the proof of significant emission reduction (see [1,2] for example). Yet, actual vehicle implementation implies frequent transients which reveal to be much more complex than steady state experimentation.

The HCCI combustion mode consists of preparing a highly diluted burnt gas/air/fuel mixture. Simultaneous ignition in the whole combustion chamber is performed and controlled. In that mode, the Burnt Gas Rate $(B G R)$ in the intake manifold plays a key role (see Fig. 2). Offsets on $B G R$ may cause misfires and additive noises. In the HCCI combustion mode it is very high (40\% or more). Accurate control of $B G R$ can be achieved by controlling the whole airpath system: intake and exhaust manifolds, EGR loop and VGT. This is the subject of the paper.

As studied in $[3,4]$, the airpath system of a turbocharged Diesel engine features coupled dynamics. The EGR acts as a discharge valve for the turbocharger. Most studies consider the following control setup: both intake and exhaust pressure are closely controlled using EGR valve and Variable Geometry Turbocharger (VGT) (see [10-12] for example). As presented in [5-7] multivariable control strategies vastly outperform decentralized mono-variable schemes. Robustness is increased since coupling is taken into account by these multivariable designs. As recalled earlier, HCCI combustion mode requires transients from small to very large $B G R$ rates to be considered. We believe that the previous approaches might not be well adapted to handle such relatively extreme situations.

The contribution of this paper is a control design for the airpath using an explicit feed-forward term. This control law arises from a motion planning open-loop control strategy. Originally, our objective is to control the masses aspirated into the cylinder. We cast it into the control of $B G R$ and pressure in the intake manifold $\left(P_{\text {int }}\right)$. The set points depend on the engine operating conditions: from low load, low pressure and high $B G R$ rate (typically $B G R=40 \%$ and $P_{\text {int }}=1$ bar) to high load, high pressure and no EGR (typically $B G R=0 \%$ and $P_{\text {int }}=2$ bar). We demonstrate the relevance of our approach on such large transients.
The paper is organized as follows. In Section 2, we detail the intake manifold model used for our study. It consists of two balance equations. In Section 4, we decouple these fully actuated dynamics by a simple motion planning strategy. This yields an open loop control law taking into account the non minimum phase behavior of the system, already underlined in [3]. In Section 3, we design an observer that is required for real implementation: a Luenberger style observer reconstructing poorly known input gains from the intake pressure measurements. Implementation details are given in Section 6. Experimental results are reported on a 4 cylinder HCCI engine in Section 6. They stress that, despite important noises on measurements, a control strategy based on a simple model can achieve fast and accurate transients. is also pointed out. Conclusions and future directions are given in Conclusion.

\section{CONTROL PROBLEM}

This macroscopic approach is sufficient for a first order $\mathrm{NO}_{x}$ reduction. Our approach to combustion control is to manage the air and burned gas masses in the cylinder $\left(M_{a i r, c y l}\right.$ and $\left.M_{b g, c y l}\right)$. In other words, we focus on the airpath system. Flows of fresh air and the Exhaust Gas Recirculation (EGR) mix into the intake manifold and are aspirated into the cylinders. In practical applications, the considered masses cannot be measured. However, equivalent variables can be considered. Controlling those two masses is equivalent to controlling the intake pressure $P_{\text {int }}$ (being an image of $\left.M_{\text {air, }, y l}+M_{b g, c y l}\right)$ and the burned gas rate $F_{\text {int }}$ (representing to ratio $\left.\frac{M_{b y, c y l}}{M_{a i r, c y l}+M_{b g, c y l}}\right)$. Setpoints are often chosen to maximize EGR in order to lower the $\mathrm{NO}_{x}$ emissions at low load. At high load, the EGR decreases the efficiency and imposes the use of low EGR. Typically, the setpoint at $1500 \mathrm{rpm}$ and high load is $\left(P_{\text {int }, s p}=2 \mathrm{bar}, F_{\text {int }, s p}=0.05\right)$ using low EGR, while at $1500 \mathrm{rpm}$ and low load setpoints under consideration are close to $\left(P_{\text {int }, s p}=1.013 \mathrm{bar}, F_{\text {int }, s p}=0.45\right)$ using high EGR.

In this context, the control problem we need to address is a large transient problem for a two outputs, two inputs system. The control inputs are the VGT actuator position $S_{v g t}\left(v_{1}\right)$ (ranging from 0 to 1 ) and the EGR valve normalized effective area $S_{\text {egr }}\left(v_{2}\right)$ (ranging from 0 to 1 ). Both are bounded. Other external inputs include the fueling rate $M_{f u e l}$ and the engine speed $N_{e}$. The underlying dynamics is also of dimension 2. The states are the outputs: $P_{\text {int }}$ and $F_{\text {int }}$.

\section{INTAKE MANIFOLD MODELLING}

Flows from the fresh air (measured by the Manifold Air Flow) and the Exhaust Gas Recirculation come into the intake manifold and are aspirated into the cylinders. For modelling, we use mass balances, ideal gas law, and consider a low time resolution ( $180^{\circ}$ TDC time scale). 
In particular, high frequency aspiration phenomena are not taken into account.

Ideal gas law in the intake manifold leads to

$$
P_{i n t} V_{i n t}=M_{i n t} R T_{i n t}
$$

Assuming that the variation of temperature is slow (i.e. $\dot{T}_{\text {int }}=0$ ), the mass balance writes

$$
\dot{P}_{i n t}=\frac{R}{V_{i n t}}\left(D_{a i r}+D_{e g r}-D_{a s p}\right)
$$

Classically (see [13] for exemple), we define the aspirated flow as

$$
D_{\text {asp }}=\eta_{v o l}\left(P_{i n t}, N_{e}\right) \frac{P_{i n t}}{R T_{i n t}} V_{c y l} \frac{N_{e}}{120}
$$

where $V_{\text {cyl }}$ is the cylinder volume. $\eta_{v o l}$ is the volumetric efficiency which is experimentally derived and, eventually, defined though a look-up table $\eta_{v o l, m a p}\left(P_{\text {int }}, N_{e}\right)$. Values vary with engine operating conditions (mainly intake pressure and engine speed).

The burned gas ratio $F_{\text {int }}$ is the fraction of burned gas in the intake manifold. It is written as

$$
F_{i n t} \triangleq 1-\frac{M_{\text {int,air }}}{M_{\text {int }}}
$$

The composition of the EGR $\left(F_{\text {egr }}\right)$ is the composition in the exhaust manifold $\left(F_{\text {exh }}\right)$ delayed by the transport through the EGR pipe. We consider that this delay is negligible, i.e. $F_{\text {egr }}=F_{\text {exh }}$. Mixing dynamics is modelled as

$$
\dot{F}_{i n t}=\frac{R T_{i n t}}{P_{\text {int }} V_{\text {int }}}\left(D_{e g r}\left(F_{\text {exh }}-F_{\text {int }}\right)-D_{\text {air }} F_{i n t}\right)
$$

The EGR flow can be expressed as

$$
D_{e g r}=S_{e g r} \frac{P_{e x h}}{\sqrt{R T_{e x h}}} \sqrt{\frac{2 \gamma}{\gamma-1}\left(p_{r}^{\frac{2}{\gamma}}-p_{r}^{\frac{\gamma-1}{\gamma}}\right)}
$$

where $p_{r}=\max \left\{\frac{P_{\text {int }}}{P_{\text {exh }}},\left(\frac{2}{\gamma+1}\right)^{\frac{\gamma}{\gamma+1}}\right\}$. These two variables describe both subsonic and choked EGR flow. In this model, numerous variables are not measured. The exhaust pressure and temperature for example are not easily available on a commercial engine. The effect of the EGR cooler is accounted for by considering

$$
D_{e g r} \triangleq \Theta_{e g r} S_{e g r}
$$

where $\Theta_{\text {egr }}$ is a variable depending on the exhaust temperature, the pressure ratio between intake and exhaust manifold, and the behavior of the cooling system. This variable needs to be estimated to evaluate the EGR flow and the composition in the intake manifold. This is the purpose of the following section.

\section{AIR PATH OBSERVER}

In this section we design an observer to estimate both the EGR flow and the BGR.

\subsection{Reference Model}

Let

$$
x=\left[P_{\text {int }} F_{\text {int }} \Theta_{\text {egr }}\right]^{T} \in \mathbb{R}^{3}
$$

be the state and $y=P_{\text {int }}$ the measurement. We note $\alpha_{\text {int }} \triangleq$ $\frac{R T_{\text {int }}}{V_{\text {int }}}$ and $\beta_{\text {int }} \triangleq \frac{1}{R T_{\text {int }}} V_{\text {cyl }} \frac{N_{e}}{120}$. Using (1) and (3), the reference dynamics reads

$$
\left\{\begin{array}{l}
\dot{x}_{1}=\alpha_{\text {int }}\left(D_{\text {air }}+x_{3} u-\eta_{\text {vol }}\left(x_{1}, N_{e}\right) \beta_{\text {int }} x_{1}\right) \\
\dot{x}_{2}=\frac{\alpha_{\text {int }}}{x_{1}}\left(F_{\text {exh }} x_{3} u-\left(D_{\text {air }}+x_{3} u\right) x_{2}\right) \\
\dot{x}_{3}=0 \\
y=x_{1}
\end{array}\right.
$$

Notations are summarized in Table 1.

TABLE 1

Variables description for the isothermal observer

\begin{tabular}{c|c||c|c}
\hline Var. & Symb. & Var. & Symb. \\
\hline$x_{1}$ & $P_{\text {int }}$ & $y$ & $P_{i n t}$ \\
\hline$x_{2}$ & $F_{i n t}$ & $\alpha_{i n t}$ & $\frac{R T_{i n t}}{V_{i n t}}$ \\
\hline$x_{3}$ & $\Theta_{e g r}$ & $\beta_{i n t}$ & $\frac{1}{R T_{i n t}} V_{c y l} \frac{N_{e}}{120}$ \\
\hline
\end{tabular}

\subsection{Observer Design}

The observer dynamics are

$$
\left\{\begin{array}{c}
\dot{\hat{x}}_{1}=\alpha_{\text {int }}\left(D_{\text {air }}+\hat{x}_{3} u-\eta_{\text {vol }}\left(y, N_{e}\right) \beta_{\text {int }} \hat{x}_{1}\right) \\
\quad-L_{1}\left(\hat{x}_{1}-y\right) \\
\dot{\hat{x}}_{2}=\frac{\alpha_{\text {int }}}{y}\left(F_{\text {exh }} \hat{x}_{3} u-\left(D_{\text {air }}+\hat{x}_{3} u\right) \hat{x}_{2}\right) \\
\dot{\hat{x}}_{3}=-L_{3}\left(\hat{x}_{1}-y\right)
\end{array}\right.
$$

with $\left(L_{1}, L_{3}\right) \in\left(\mathbb{R}^{+} \backslash\{0\}\right)^{2}$. One can notice that (5) is a copy of (4) with additive tracking terms. Unknowns are partially substituted with output measurement. The state-error is $\tilde{x} \triangleq$ $x-\hat{x}$. Classically, the error dynamics are

$$
\left\{\begin{array}{l}
\dot{\tilde{x}}_{1}=\alpha_{i n t}\left(\tilde{x}_{3} u-\eta_{v o l}\left(y, N_{e}\right) \beta_{i n t} \tilde{x}_{1}\right)-L_{1} \tilde{x}_{1} \\
\dot{\tilde{x}}_{2}=\frac{\alpha_{i n t}}{y}\left(F_{\text {exh }} \tilde{x}_{3} u-\left(D_{\text {air }}+\hat{x}_{3} u\right) \tilde{x}_{2}+\left(F_{\text {exh }}-x_{2}\right) \tilde{x}_{3}\right) \\
\dot{\tilde{x}}_{3}=-L_{3} \tilde{x}_{1}
\end{array}\right.
$$

Tuning parameters are chosen as follows:

$$
\left\{\begin{array}{l}
L_{1}=\left(l_{1}-\eta_{v o l}\left(y, N_{e}\right)\right) \alpha_{i n t} \beta_{i n t} \\
L_{3}=l_{3} \alpha_{i n t} u
\end{array}\right.
$$


where $l_{1}$ and $l_{3}$ are positive constants. With this choice, the error system can be written under the triangular form (6)-(7)

$$
\left[\begin{array}{c}
\dot{\tilde{x}}_{1} \\
\tilde{\tilde{x}}_{3}
\end{array}\right]=A_{\text {int }}\left[\begin{array}{c}
\tilde{x}_{1} \\
\tilde{x}_{3}
\end{array}\right]
$$

where

$$
A_{\text {int }} \triangleq\left[\begin{array}{cc}
-l_{1} \alpha_{i n t} \beta_{i n t} & \alpha_{i n t} u \\
-l_{3} \alpha_{i n t} u & 0
\end{array}\right]
$$

and

$$
\begin{gathered}
\dot{\tilde{x}}_{2}=\frac{\alpha_{\text {int }}}{y}\left(F_{\text {exh }} \tilde{x}_{3} u-\left(D_{\text {air }}+\hat{x}_{3} u\right) \tilde{x}_{2}\right) \\
+\frac{\alpha_{\text {int }}}{y}\left(F_{\text {exh }}-x_{2}\right) \tilde{x}_{3}
\end{gathered}
$$

Tuning and convergence of the proposed observer around fixed operating point has been investigated in [14] resulting in the following proposition.

Proposition 1 For any fixed operating point, i.e. constant values of $F_{e x h}, T_{i n t}, N_{e}$, and $u>0$, the state of observer (5) exponentially converges towards the state of system (4).

\section{AIR PATH CONTROL FEEDFORWARD}

Now, we have an estimation of the EGR flow and the BGR in the intake manifold. Thanks to that well defined change of variables, the control inputs are the EGR flow $D_{\text {egr }}$ and the fresh air flow $D_{\text {air }}$. The reference model for the control is written as

$$
\left\{\begin{array}{l}
\dot{x}_{1}=\alpha_{i n t}\left(u_{1}+u_{2}-\eta_{v o l}\left(x_{1}, N_{e}\right) \beta_{i n t} x_{1}\right) \\
\dot{x}_{2}=\frac{\alpha_{i n t}}{x_{1}}\left(F_{\text {exh }} u_{2}-\left(u_{1}+u_{2}\right) x_{2}\right)
\end{array}\right.
$$

We propose a motion planning control strategy which relies on the computation of transient trajectories for the airpath dynamics (8). This strategy is detailed in Figure 2. It comprises 4 sub procedures: setpoint computations through static maps (first two blocks in Fig. 2), trajectory generation, model inversion, and saturation of open-loop control values. We now detail these in the next subsection, we actually prove convergence of the airpath system when using this strategy.

\subsection{Set Points}

The driver's request considered here is the accelerator position. First, taking into account the gear box configuration, this request is turned into a torque control objective under the form of an IMEP (Indicated Mean Effective Pressure) set point. Then, the set points for the intake pressure and the BGR (noted $x^{\mathrm{sp}}$ in Fig. 2) are inversely given by experimentally calibrated static maps on the $\left(\mathrm{IMEP}^{\mathrm{sp}}, N_{e}\right)$ operating range. The engine speed $N_{e}$ is not modelled but directly measured. The $x^{\mathrm{sp}}=\left(x_{1}^{\mathrm{sp}}, x_{2}^{\mathrm{sp}}\right)$ vector is defined as

$$
x_{1}^{\mathrm{sp}}=f_{\mathrm{pressure}}\left(\mathrm{IMEP}^{\mathrm{sp}}, N_{e}\right) \text { and } x_{2}^{\mathrm{sp}}=f_{\mathrm{bgr}}\left(\operatorname{IMEP}^{\mathrm{sp}}, N_{e}\right)
$$

\subsection{Motion Planning}

Because IMEP ${ }^{\mathrm{sp}}$ is arbitrarily specified by the driver, $t \mapsto$ $x_{1}^{\mathrm{sp}}(t)$ and $t \mapsto x_{2}^{\mathrm{sp}}(t)$ may not be smooth nor monotonous. These signals must be filtered to correspond to feasible trajectories of (8). This can be done by many methods. Here, we propose the following approach that, besides other interesting properties, is easy to handle in the convergence analysis process. It addresses only the case of transients from one steady state to another. From a current steady state $\underline{x}$ to a target $\bar{x}$ we use an interpolation formula (9). Coordinatewise this defines $x_{1}^{\mathrm{mp}}$ and $x_{2}^{\mathrm{mp}}$. Let

$$
\phi(t, T)=\left\{\begin{array}{cl}
0 & \text { for } 0 \geq t \\
\left(\frac{t}{T}\right)^{2}\left(3-2 \frac{t}{T}\right) & \text { for } 0 \leq t \leq T \\
1 & \text { for } T \leq t
\end{array}\right.
$$

Note two positive constants $T_{1}$ and $T_{2}$. The considered interpolation is

$$
\left\{\begin{array}{l}
x_{1}^{\mathrm{mp}}(t)=\underline{x}_{1}+\left(\bar{x}_{1}-\underline{x}_{1}\right) \phi\left(t, T_{1}\right) \\
x_{2}^{\mathrm{mp}}(t)=\underline{x}_{2}+\left(\bar{x}_{2}-\underline{x}_{2}\right) \phi\left(t, T_{2}\right)
\end{array}\right.
$$

\subsection{Model Inversion}

System (8) is fully actuated and invertible. Thus, an analytic expression of the input can be derived from the state variables and their first derivatives histories. In fact,

$$
\left\{\begin{array}{c}
u_{1}+u_{2}=\eta_{v o l}\left(x_{1}, N_{e}\right) \beta_{i n t} x_{1}+\frac{1}{\alpha_{i n t}} \dot{x}_{1} \\
-x_{2} u_{1}+\left(F_{e x h}-x_{2}\right) u_{2}=\frac{1}{\alpha_{i n t}} \dot{x}_{2} x_{1}
\end{array}\right.
$$

This rewrites as

$$
\left\{\begin{array}{l}
u_{1}=f_{1}(x, \dot{x}) \\
u_{2}=f_{2}(x, \dot{x})
\end{array}\right.
$$

with

$$
\left\{\begin{aligned}
f_{1}(x, \dot{x})=\frac{1}{F_{e x h}} & \left(\frac{F_{e x h}-x_{2}}{\alpha_{\text {int }}} \dot{x}_{1}-\frac{1}{\alpha_{\text {int }}} \dot{x}_{2} x_{1}\right. \\
& \left.+\left(F_{\text {exh }}-x_{2}\right) \eta_{\mathrm{vol}}\left(x_{1}, N_{e}\right) \beta_{\text {int }} x_{1}\right) \\
f_{2}(x, \dot{x})=\frac{1}{F_{e x h}}\left(\frac{1}{\alpha_{\text {int }}} x_{2} \dot{x}_{1}\right. & \\
& \left.+\eta_{\mathrm{vol}}\left(x_{1}, N_{e}\right) \beta_{\text {int }} x_{2} x_{1}+\frac{1}{\alpha_{\text {int }}} \dot{x}_{2} x_{1}\right)
\end{aligned}\right.
$$

In these last expressions, $F_{\text {exh }}, \alpha_{i n t}, N_{e}$, and $\beta_{i n t}$ are all given by sensors measurements. The unique open-loop control law $\left(u_{1}^{\mathrm{mp}}, u_{2}^{\mathrm{mp}}\right)$ corresponding to any desired $\left(x_{1}^{\mathrm{mp}}, x_{2}^{\mathrm{mp}}\right)$ trajectory (defined by formulas (10)) is

$$
\left\{\begin{array}{l}
u_{1}^{\mathrm{mp}}=f_{1}\left(x_{1}^{\mathrm{mp}}, \dot{x}_{1}^{\mathrm{mp}}, x_{2}^{\mathrm{mp}}, \dot{x}_{2}^{\mathrm{mp}}\right) \\
u_{2}^{\mathrm{mp}}=f_{2}\left(x_{1}^{\mathrm{mp}}, \dot{x}_{1}^{\mathrm{mp}}, x_{2}^{\mathrm{mp}}, \dot{x}_{2}^{\mathrm{mp}}\right)
\end{array}\right.
$$




\subsection{Input Constraints}

There are several constraints that need to be considered. Input signals $\left(u_{1}, u_{2}\right)$ and the aspirated flow $\left(u_{1}+u_{2}\right)$ must be positive and strictly positive, respectively, because they correspond to input flows. Also, the inputs must not cause misfires (usually due to high EGR). A simple strategy can address this issue. Conservatively, misfire avoidance can be guaranteed provided the following input constraints are satisfied

$$
C(u) \triangleq F_{\text {exh }} \frac{u_{2}}{u_{2}+u_{1}} \leq \bar{C}<1
$$

$C(u)$ is equivalent to the BGR at steady state. Adding the previously discussed positiveness of input flows, the set of admissible inputs is defined as

$$
\begin{array}{r}
\mathcal{U} \triangleq\left\{\left(u_{1}, u_{2}\right) \in(\mathbb{R})^{2} / u_{1}+u_{2} \geq u_{\min }, u_{2}\right. \\
\text { and } \left.F_{\text {exh }} \frac{u_{2}}{u_{2}+u_{1}} \leq \bar{C}\right\}
\end{array}
$$

and we note $\partial \mathcal{U}$ its boundary. We define the constrained control as

$$
u^{\mathrm{ol}}(t) \triangleq \arg \left(\min _{u \in \mathcal{U}}\left(u_{1}-u_{1}^{\mathrm{mp}}(t)\right)^{2}+\left(u_{2}-u_{2}^{\mathrm{mp}}(t)\right)^{2}\right)
$$

where $u_{1}^{\mathrm{mp}}(t)$ and $u_{2}^{\mathrm{mp}}(t)$ are defined by (14). A representation of the input constraints in the plane is given in Figure 1. In other words, for all $t \in \mathbb{R}^{+}, u^{\mathrm{ol}}(t)$ is the projection of $u^{\mathrm{mp}}(t)=\left(u_{1}^{\mathrm{mp}}, u_{2}^{\mathrm{mp}}\right)(t)$ onto the set $\mathcal{U}$. In (15), both the cost function and the admissible set $\mathcal{U}$ are convex. Thus, there always exists a uniquely defined minimum $u^{\text {ol }}$. In fact, this solution can be analytically computed in a straightforward way (by enumerating 6 possible solutions), which is compatible with our real-time control application requirements.

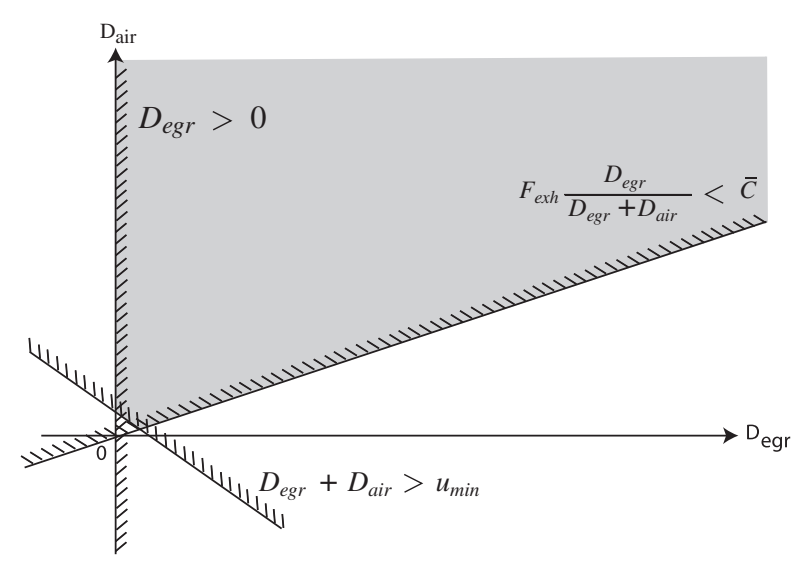

Figure 1

Input constraints in the plan $\left(u_{1}=D_{\text {air }}, u_{2}=D_{\text {egr }}\right)$, the admissible set $\mathcal{U}$ is represented in grey.

\subsection{Convergence and Tuning of the Constrained Motion Planning}

We now prove that the proposed control strategy actually converges. The main focus is on the impact of input saturations as previously described.

It is assumed that the volumetric efficiency slowly varies w.r.t. the intake pressure $x_{1}$. Denoting $h\left(x_{1}, N_{e}\right) \triangleq$ $\eta_{v o l}\left(x_{1}, N_{e}\right) x_{1}$, we suppose that there exists a strictly positive constant $\bar{h}$ such that for all $\left(x_{1}, N_{e}\right)$ in $\mathbb{R}^{+} \times[500,4500]$,

$$
\frac{\partial h}{\partial x_{1}}\left(x_{1}, N_{e}\right) \geq \bar{h}>0
$$

Experimentally, this assumption is actually easy to validate.

\subsubsection{Exponential Stability}

In [15], stability was studied by exploiting the cascade structure of equations (8) leading to the following proposition.

Proposition 2 Consider system (8). For any constant input $u=\left(\bar{u}_{1}, \bar{u}_{2}\right)$, the state $x$ exponentially converges toward $z \triangleq$ $\left(z_{1}, z_{2}\right)$ where $h\left(z_{1}, N_{e}\right)=\bar{u}_{1}+\bar{u}_{2}$ and $z_{2}=F_{\text {exh }} \frac{\bar{u}_{2}}{\bar{u}_{1}+\bar{u}_{2}}$. A way to asymptotically reach $\bar{x}=\left(\bar{x}_{1}, \bar{x}_{2}\right)$ is to use a constant input $\bar{u}$ as defined in (16).

Conversely, it is possible to tune the input values to reach a desired set point $\bar{x}=\left(\bar{x}_{1}, \bar{x}_{2}\right)$. For that purpose, one should use

$$
\left\{\begin{array}{l}
\bar{u}_{1}=\frac{F_{e x h}-\bar{x}_{2}}{F_{e x h}} \eta_{\mathrm{vol}}\left(\bar{x}_{1}, N_{e}\right) \beta_{i n t} \bar{x}_{1} \\
\bar{u}_{2}=\frac{1}{F_{e x h}} \eta_{\mathrm{vol}}\left(\bar{x}_{1}, N_{e}\right) \beta_{\text {int }} \bar{x}_{1} \bar{x}_{2}
\end{array}\right.
$$

This proposition is the key to understanding our approach. Provided chosen control values are feasible (i.e. belong to $\mathcal{U}$ ), it is sufficient to use them as the step inputs in the airpath system to asymptotically reach the desired set point. Now, as we mentioned it in the Introduction, our goal is to provide more efficient transients. Ideally, we would like to have soft landings and fast transients. If the proposed control (14) are feasible, then $u^{\mathrm{mp}}(t)=u^{\mathrm{ol}}(t)$ for all $t \in \mathbb{R}^{+}$and, neglecting possible perturbations, the transient is perfectly achieved. Yet, if the motion planning strategy is, at times, inconsistent with the input constraints, then $u^{\mathrm{mp}}(t) \neq u^{\mathrm{ol}}(t)$ during the transient. In any cases, for large values of $t\left(t \geq \max \left\{T_{1}, T_{2}\right\}\right.$ as used in (9)), both coincide again and equal the feasible final input values. Ultimately, the system converges. The motion planning strategy can only improve transients when the computed input values are feasible, at least over some reasonably long period of the transient interval.

We now perform some analysis that allows us to guarantee this desired feasibility. From this, we can derive guidelines to tune parameters $T_{1}$ and $T_{2}$. 


\subsubsection{Transient Tuning}

In [15], we showed that for large enough values for $T_{1}$ and $T_{2}$, the control values do not violate the constraints. Moreover, we proved that for small enough values for $T_{1}$ and $T_{2}$, the control values do violate the constraints. Constructive choices for $T_{1}$ and $T_{2}$ in both cases are also given. Consider the motion planning strategy (14) aiming at steering the system from steady state $(\underline{x}, \underline{u}) \in \mathcal{U}$ to $(\bar{x}, \bar{u}) \in \mathcal{U}$. From this performance analysis, we can propose the following tuning methodology for $T_{1}$ and $T_{2}$.

1. We choose $\epsilon_{1}>0$ and pick $T_{1}=\frac{2}{\epsilon_{1}} \frac{\left|\bar{x}_{1}-\underline{x}_{1}\right|}{\alpha_{\text {int }}}$ to account for turbocharger inertia (which drives the air flow dynamics).

2. We choose $\epsilon_{2}>0$ and use

$$
\begin{aligned}
T_{2}=\max \left\{T_{2}\right. & \in\left[T_{m}\left(T_{1}\right), T_{M}\left(T_{1}, \epsilon_{2}\right)\right] / \\
\forall t & \left.\in \mathbb{R}^{+} \operatorname{dist}\left(u^{\mathrm{mp}}(t), \mathcal{U}\right)<\epsilon_{2}\right\}
\end{aligned}
$$

where

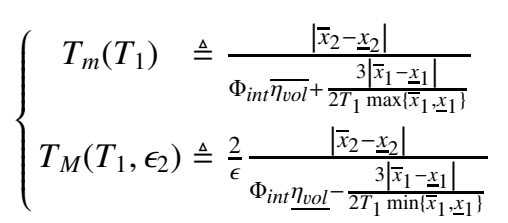

These rules guarantee that the constraints are not violated by more than $\max \left\{\epsilon_{1}, \epsilon_{2}\right\}$.

\section{AIR PATH CONTROL FEEDBACK}

Fast PID controllers are added to the structure to provide further accuracy and robustness. The goal of the feedback is to control the EGR flow $D_{\text {egr }}$ and the air flow $D_{\text {air }}$ toward the reference setpoints $D_{e g r}^{\mathrm{ol}}$ and $D_{a i r}^{\mathrm{ol}}$. The main purpose of the feedforward is to give a feasible and continuous setpoint for the feedback action.

\subsection{EGR Position to $D_{\text {egr }}$}

Increasing the EGR position corresponds to opening the EGR valve and, thereby, increasing the EGR flow $D_{\text {egr }}$. The flow response is almost instantaneous, the EGR valve opening control the EGR flow $D_{\text {egr }}$. More precisely, a PI controller with an anti-wind up action on the normalized EGR flow $\left(\frac{D_{\text {egr,sp }}-\hat{D}_{\text {egr }}}{\hat{\Theta}_{\text {egr }}}\right)$ is used on the EGR valve.

\subsection{EGR/VGT to $D_{\text {air }}$}

Increasing the VGT position, i.e. opening the guide vanes, leads to a greater restriction of the exhaust gas flow and then to a decrease of the exhaust manifold pressure.

When the EGR valve is wide open (at low speed and low load e.g.), increasing the VGT results in a decreased EGR flow $D_{\text {egr }}$ which in turn increases the air flow $D_{\text {air }}$.
TABLE 2

Variation of air/egr flows depending on VGT

\begin{tabular}{lll}
\hline Low EGR & & \\
\hline Decreasing VGT & $\Rightarrow$ & High increasing $D_{\text {air }}$ \\
& $\Rightarrow$ & Low increasing $D_{\text {egr }}$ \\
\hline High EGR & & \\
\hline Decreasing VGT & $\Rightarrow$ & Low decreasing $D_{\text {air }}$ \\
& $\Rightarrow$ & Low increasing $D_{\text {egr }}$ \\
\hline
\end{tabular}

When the EGR valve is almost closed, most of the exhaust gas must pass through the turbine. Increasing the VGT decreases the compressor power and, then, increases the air flow. In this case, the VGT acts as a conventional wastegate, i.e. the VGT directly controls the turbocharger speed and the air flow $D_{\text {air }}$. Therefore, the steady-state gain from VGT to $D_{\text {air }}$ undergoes a sign change depending on the operating point. Since it is uncertain where the sign change occurs, the VGT should not be used to track $D_{\text {air }}$ setpoints in a decentralized strategy. Rather, the strategy should use the EGR flow information. The variation of intake pressure is monotonic w.r.t. VGT. Depending on the opening of the EGR valve, i.e. depending on the EGR flow, the variation of pressure is very different. Indeed, in practice, with very high EGR rate, the variation of the VGT slightly impact the intake pressure. A tedious calibration work is a solution.

\section{EXPERIMENTAL RESULTS}

\section{Implementation}

The global control scheme is summarized in Figure 2. The air path observer block (Block (A)) is the implementation of the observer described in Section 3. This block gives an estimation of the BGR and the EGR flow. The motion planning block (Block (B)) is the implementation of the motion planning open loop control strategy described in Section 4. Fast PID controllers are added to the structure (Block (C)) to provide further accuracy and robustness. Their implementation is described in Section 5.

The control was tested on torque trajectory at fixed engine speed $(1500 \mathrm{rpm})$. This torque trajectory mixes HCCI combustion mode (for IMEP $s p<7$ bar) and conventional combustion mode. The same calibration was kept on all the torque trajectory. The IMEP demand is given in Figure 3. On IMEP transient in HCCI mode, it occurs that we want to increase both BGR and intake pressure. This is the case when IMEP of the system starts at 2 bar and eventually reaches 5 bar (from $t=102 \mathrm{~s}$ to $t=112 \mathrm{~s}$ ). This transient aims higher intake pressure and BGR setpoints. Starting and ending operating points are both in HCCI combustion mode. Let us focus on Figures 6 and 7 (from $t=102 \mathrm{~s}$ to $t=112 \mathrm{~s})$. By contrast with all decentralized controllers, we notice on that our controller takes into account the well 


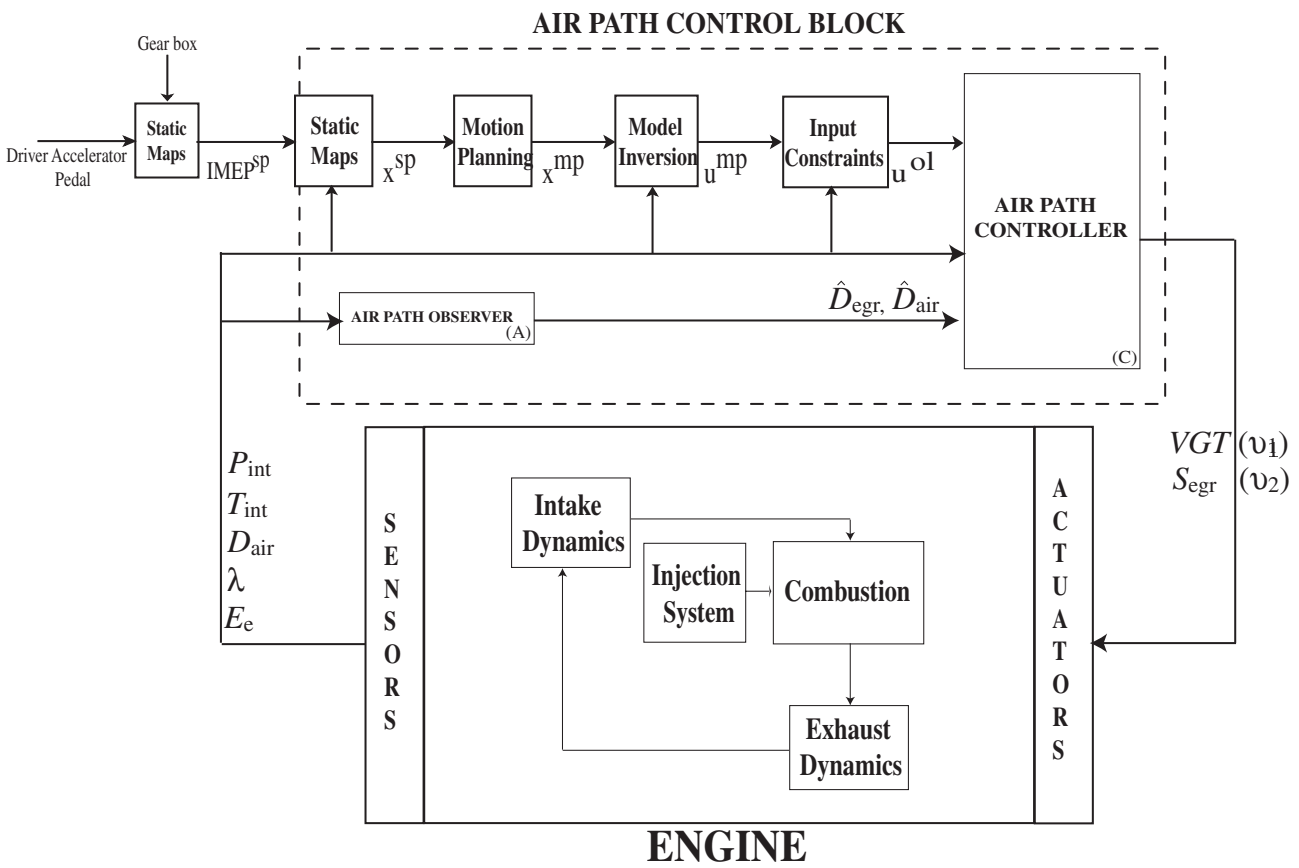

Figure 2

Control Scheme.

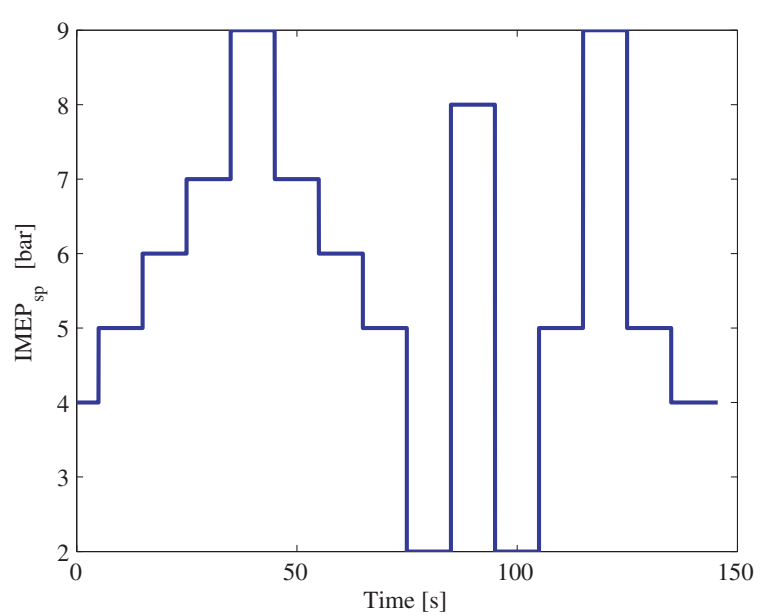

Figure 3

Torque trajectory at constant engine speed $(1500 \mathrm{rpm})$.

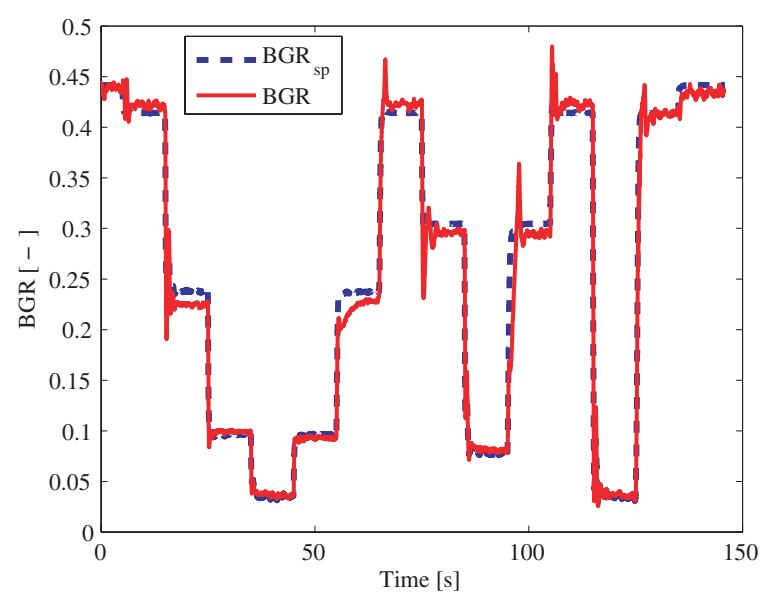

Figure 4

Experimental results on a torque trajectory at constant engine speed (1500 rpm): BGR histories. Dashed: set point, solid: closed-loop trajectory. known non minimum phase behavior of the system reported in [3]. More precisely, one can check that the main contribution to this is due to the open-loop controller (the closed loop control histories being very close to it). When the EGR valve opens, the flow increases. This leads to a pressure rise in the intake manifold. Meanwhile, the exhaust pipe acts as a discharge for the VGT. Its opening lowers the EGR supplied to the turbocharger yielding a significant drop of the exhaust manifold flow. The turbocharger slows down, which eventually causes the decrease of the intake manifold pressure. This phenomenon is delayed and slowed down by the turbocharger inertia. Simple ramps and/or steps would fail to let the system reach the desired setpoint. With the proposed control strategy, the model takes into account this 


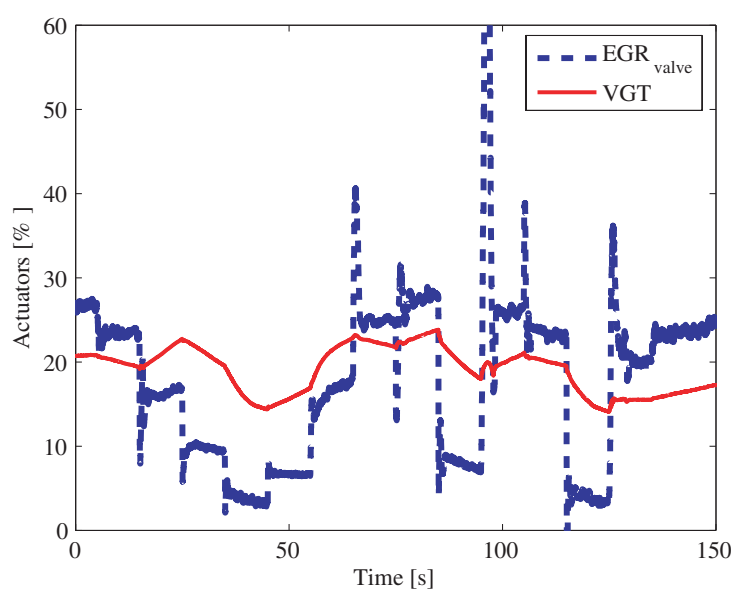

Figure 5

Experimental results on a torque trajectory at constant engine speed (1500 rpm): actuators histories. Dashed: EGR valve, solid: VGT.

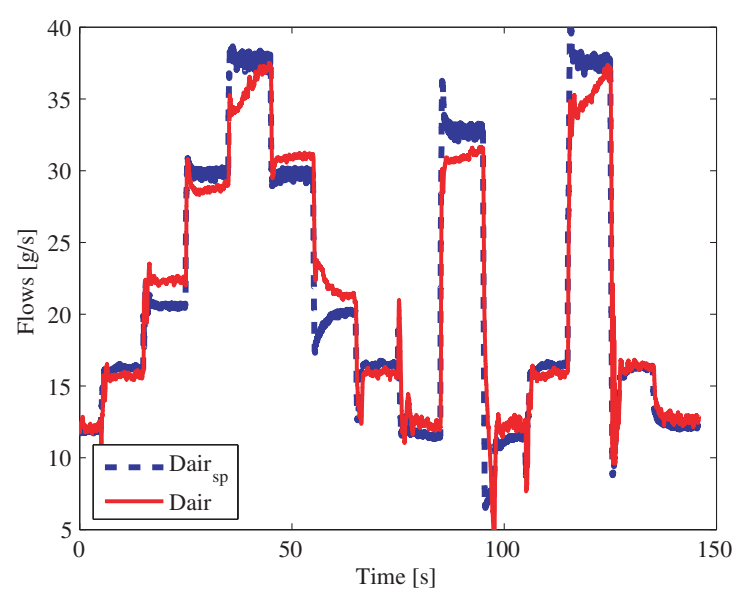

Figure 6

Experimental results on a torque trajectory at constant engine speed (1500 rpm): air flow histories. histories. Dashed: set point, solid: closed-loop trajectory.

complex behavior. The motion planning efficiently drives the system to its setpoint.

The air and EGR flows setpoints and closed-loop trajectory are presented on Figures 6 and 7. As in the following cases, the EGR flow is almost perfectly tracked, the air flow tracking is good but a little bit slow during large transients due to the turbocharger inertia. In summary, the results are good, even with a reasonably large transient. We are able to follow the planned trajectory. High pressure setpoints are more difficult to reach due to the turbocharger inertia and friction. However, it is possible to relax the intake pressure tracking because, for pollutant reduction purposes, only BGR needs to be closely controlled provided a limited Air-

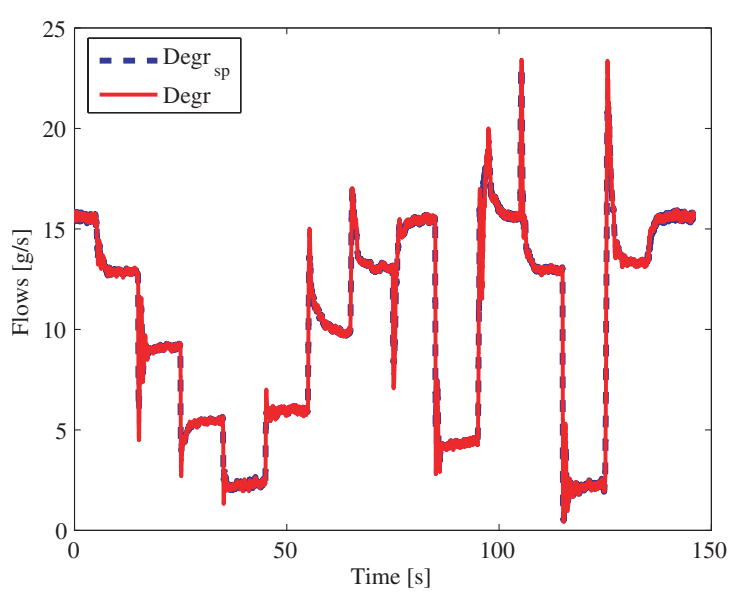

Figure 7

Experimental results on a torque trajectory at constant engine speed (1500 rpm): EGR flow histories. histories. Dashed: set point, solid: closed-loop trajectory.

Fuel Ratio is guaranteed. The errors on the intake pressure will only lead to a very small error on the torque production. Nevertheless, on a vehicle, this problem will not appear because as the torque production increases, in response, the engine speed and the turbocharger speed increases. This phenomenon is expected to be reduced in real-vehicle applications.

\section{CONCLUSIONS AND FUTURE WORK}

\section{Conclusion}

The presented work demonstrates the relevance of motion planning in the control of the -coupled- airpath dynamics of turbocharged Diesel engines using Exhaust Gas Recirculation. For the HCCI combustion mode, very large rates of burned gas need to be considered and we have proven on realistic test-bench cases that the proposed approach can handle such situations. Despite strong coupling and possible nonminimum phase dynamics, the airpath dynamics has nice properties that make it easy to steer through our control strategy. Its triangular form yields exponential convergence over a wide range of setpoints. Moreover, we showed that input constraints can be added without jeopardizing convergence.

\section{Toward general airpath control}

The presented work is validated experimentally on a HCCI engine with a high pressure EGR circuit and a Variable Geometry Turbocharger. The next step is the extension of the control strategy to other engine configurations, e.g. considering low pressure EGR circuit, waste-gate, two-stage 
turbocharger, intake throttle... The control strategy can be kept. The observer will be the same (Block (A) in Fig. 2), and so will the motion planning. The main modification will be to decentralize the control in order to use all the actuators for the control of the air flow $D_{\text {air }}$ and the EGR flow $D_{e g r}$.

\section{REFERENCES}

1 Kahrstedt, J., Behnk, K., Sommer, A., and, T. Wormbs (2003). Combustion processes to meet future emission standards. In Motortechnische Z., 1417-1423.

2 Hultqvist, A., Engdar, U., Johansson, B., and Klingmann, J. (2001). Reacting boundary layers in a homogeneous charge compression ignition (HCCI) engine. In Proc. of the SAE Conference, number 2001-01-1032, .

3 Kolmanovsky, I., Stefanopoulou, A., Moraal, P., and van Nieuwstadt, M. (1997). Issues in modelling and control of intake flow in variable geometry turbocharged engines. In Proc. of the 18th IFIP Conference on System Modelling and Optimization, 1997.

4 Kao, M., and Moskwa, J. (1995). Turbocharged Diesel engine modelling for nonlinear engine control and estimation. J. Dyn. Syst. - T. ASME, 117.

5 van Nieuwstadt, M. and Moraal, P. and Kolmanovsky, I. and Stefanopoulou, A. and Wood, P. and Criddle, M. (1998). Decentralized and multivariable designs for EGR-VGT control of Diesel engine. Proc. of the 2nd IFAC Workshop on Advances in Automotive Control.

6 Stefanopoulou, A. and Kolmanovsky, I. and Freudenberg, J.(2000). Control of Variable Geometry Turbocharged Diesel Engines for Reducted Emissions. IEEE T. Contr. Syst. T., 8, 733-745.

7 van Nieuwstadt, M., Kolmanovsky, I., Moraal, P., Stefanopoulou, A., Janković, M. (2000). Experimental Compar- ison of EGR-VGT Control Schemes for a High Speed Diesel Engine. Control Syst. Mag., 20, 63-79.

8 Jung, M. and Glover, K. (2003) Control-Oriented Linear Parameter-Varying Modelling of a Turbocharged Diesel Engine. In Conference on Control Application.

9 Jung, M. and Glover, K. (2005) Comparison of Uncertainty Parameterisations for H-infinity Robust Control of Turbocharged Diesel Engines. Control Eng. Pract., 13, 15-25.

10 Ammann, M., Fekete, N., Guzzella, L., and Glattfelder, A. (2003). Model-based control of the VGT and EGR in a turbocharged common-rail Diesel engine: theory and passenger car implementation. In Proc. of the SAE Conference, number 2003-01-0357.

11 Janković, M., and Kolmanovsky, I. (1998). Robust nonlinear controller for turbocharged Diesel engine. Proc. of the American Control Conference.

12 Janković, M., and Kolmanovsky, I. (2000). Constructive Lyapounov Control Design for Turbocharged Diesel Engines. IEEE T. Contr. Syst. T., 8, 288-299.

13 Heywood, J. (1988). Internal Combustion Engine Fundamentals. McGraw-Hill, Inc, 1988.

14 Chauvin, J., Corde, G., Vigild, C., Petit, N., and Rouchon, P. (2006). Air path estimation on Diesel HCCI engine. In Proc. of the SAE Conference, number 2006-01-1085.

15 Chauvin J., Corde, G., and Petit, N. (2006). Constrained motion planning for the airpath of a Diesel HCCI engine. In Proc. of the IEEE Conf. Decision and Control.

Final manuscript received in October 2006 